\title{
EDITORIAL
}

\section{Gracias y Feliz Navidad}

\section{Thank you very much and merry christmas}

\author{
Autoras: \\ Maciá Soler, Loreto \\ Carratalá Munuera, Concepción
}



1 apartado editorial del número 18 de la revista RECIEN consiste en un mensaje de las editoras de la revista dirigido a lectores y colaboradores, además de un anuncio de interés para sus seguidores.

En primer lugar, GRACIAS por leer RECIEN y enviar sus trabajos que con- siguen poco a poco mejorar la calidad de la revista.

Durante 2019, la sociedad científica de Enfermería SCELE y la sociedad española de Enfermería en Neurociencias unieron sus esfuerzos, para tratar de hacer llegar a un mayor número de lectores los trabajos publicados y esto

Puede citar este artículo como: Maciá Soler, L.; Carratalá Munuera, C. Gracias y Feliz Navidad. RECIEN. 2019; 18. https://doi.org/10.14198/recien.2019.18.01

Este trabajo se publica bajo una licencia de Creative Commons Reconocimiento 4.0 Internacional (CC BY 4.0) 
es un acontecimiento digno de comentar.

Las publicaciones RECIEN, están inmersas en un proceso de revisión por pares, que garantizan la independencia y rigor de los resultados y la revista forma parte de la colección de revistas de la plataforma OJS de la Universidad de Alicante y si bien, podemos observar el escaso número de artículos publicados por cada ejemplar, también es cierto que la revista es relativamente joven y que no ha dejado de publicarse con la periodicidad prevista, además de asegurar que, el número de trabajos que se reciben se va incrementando progresivamente.

Gracias a todo el equipo científico que, con sus revisiones, garantizan que se cumplan los objetivos de la revista.

Anunciarles que, la sociedad científica SCELE celebrara su X congreso bianual el próximo mes de Mayo de 2020 en la Universidad de Alicante y las comunicaciones que se acepten tendrán su apartado de publicación en la sección congresos de la revista, como ha sucedido en ediciones anteriores. En la pagina web https://www.scele.org/ pueden consultar los aspectos concretos del congreso.

Les invitamos a seguir participando y aportando experiencias de cuidados y resultados de sus investigaciones para que a través de RECIEN se pueda mejorar el conocimiento enfermero, que sin duda contribuirá a mejorar la vida de las personas que reciben servicios sanitarios y socio-sanitarios de Enfermería.

Para finalizar, deseamos a todos/as nuestros lectores una Feliz Navidad y que 2020 les traiga lo mejor.

Saludos cordiales 